\title{
Interfacially-adsorbed particles enhance the self-propulsion of oil droplets in aqueous surfactant
}

Seong Ik Cheon ${ }^{1}$, Leonardo Batista Capaverde Silva ${ }^{2}$, Aditya S. Khair ${ }^{3}$, Lauren D. Zarzar*1,2,4

1. Department of Chemistry, The Pennsylvania State University, University Park, PA 16802

2. Department of Materials Science and Engineering, The Pennsylvania State University, University Park, PA 16802

3. Department of Chemical Engineering, Carnegie Mellon University, Pittsburgh, PA 15213

4. Materials Research Institute, The Pennsylvania State University, University Park, PA, 16802

*Correspondence to 1dz4@psu.edu

TOC: The adsorption of solid particles on the surface of solubilizing oil droplets can significantly enhance the droplets' self-propulsion speeds.

\begin{abstract}
Understanding the chemo-mechanical mechanisms that direct the motion of self-propulsive colloids is important for the development of active materials and exploration of dynamic, collective phenomena. Here, we demonstrate that the adsorption of solid particles on the surface of solubilizing oil droplets can significantly enhance the droplets' self-propulsion speeds. We investigate the relationship between the self-propulsion of bromodecane oil droplets containing silica particles of varying concentration in Triton X-100 surfactant, noting up to order of magnitude increases in propulsion speeds. Using fluorescently labeled silica, we observe packing of the particles at the oil-water interfaces of the rear pole of the moving droplets. For bromodecane oil droplets in Triton X-100, the highest droplet speeds were achieved at approximately $40 \%$ particle surface coverage of the droplet interface. We propose a framework to rationalize this trend wherein the adsorption of particles at the oil-water interface imparts asymmetry to the rates of oil solubilization rates across the droplet surface, resulting in a non-monotonic dependence of propulsion speed on surface coverage. This mechanism is generalizable, and we demonstrate propulsion enhancement in ionic surfactants and different oil droplet compositions. Droplets that are solubilizing but non-active could also be induced to self-propel in the presence of interfacially adsorbed particles. Thus, droplet speeds can be significantly modulated through the simple addition of particles to displace oil-water interfacial area, providing a straightforward route to tune active droplet dynamics.
\end{abstract}

\section{Introduction}

Understanding and developing chemo-mechanical mechanisms to direct the motion of colloids is a growing interest area in the field of active matter ${ }^{1,2}$. An important consideration when designing chemotactic active colloids is the mechanism by which asymmetric forces will be generated and applied to direct the particle motion. Perhaps the most common approach is to create Janus particles wherein the asymmetry is permanently built into the particle, such as having sides of differing surface chemistry or anisotropic shape ${ }^{3}$. However, isotropic colloids, such as spherical liquid droplets, can also propel when exposed to chemical gradients in the surroundings ${ }^{4}$. In the case of active droplets, motion is typically driven by interfacial tension gradients and Marangoni flows induced by interfacial reactions ${ }^{5,6}$ or by micelle-mediated solubilization, a process wherein 
the droplet contents are transferred into the continuous micellar phase ${ }^{7}$. It has been proposed that the solubilizate-surfactant interactions and the "filling" of the surfactant micelles correspond to increased interfacial tensions at the droplet surface, and thus droplets propel towards regions of "empty" micelles ${ }^{2,8,9}$. For an isotropic droplet to move via chemotaxis, asymmetry in the chemical gradient across the droplet surface must be maintained, such as by feedback processes involving advective transport dominating over diffusion (e.g. high Péclet number) ${ }^{10}$, an externally applied chemical gradient ${ }^{9,11}$ or the presence of another nearby droplet that modifies the chemical gradient symmetry $^{8,12}$. Exploring mechanisms by which to impose asymmetry into active colloids is an important step towards controlling properties such as propulsion speed, sensitivity, directionality, and energy efficiency.

In this work, we explore the effect of particles adsorbed at droplet oil-water interfaces on the self-propulsive behaviors of solubilizing oil droplets in nonionic and ionic surfactant solutions. It is well known that particles can adsorb to liquid-liquid interfaces, such as in Pickering emulsions ${ }^{13,14}$. We demonstrate that interfacial adsorption of particles can lead to significant increases in droplet speed, often by over an order magnitude, compared to self-propulsion of solubilizing droplets without particles. We visualized fluorescently-labeled silica particles during the droplet propulsion and determined that the particles pack together to create a cap on the rear pole of the droplet. The degree of surface coverage by this particle cap influenced the droplet speed. Bromodecane droplets in Triton X-100 surfactant exhibited the highest speed enhancement when silica particles covered roughly $40 \%$ of the droplet surface. Droplets which solubilize but do not self-propel, such as bromooctane in $0.5 \mathrm{wt} \%$ Triton X-100, can also be induced to rapidly swim via the addition of surface-active particles. We propose that the particles, which displace oil-water interfacial area, reduce the rate of oil solubilization at the particle cap and create enhanced asymmetry in the distribution of solubilized oil and interfacial tension gradients that drive droplet motion by Marangoni flow. The simple addition of particles to droplet surfaces thus expands our ability to not only tune droplet propulsion speeds, but also broadens the chemical compositions which can be used to create active droplets. These advances may inspire new design approaches for active colloidal swimmers.

\section{Experimental}

Materials: Fumed silica particles were generously provided by Wacker Chemie (products HDK S13, HDK H13L, HDK H20RH). The S13 silica had no surface modification, H13L had $50 \%$ surface coverage of dimethylsiloxy groups, and H20RH silica had 75\% surface coverage of long ( $\sim 16$ carbons on average) saturated hydrocarbon chains. These fumed silica particles are amorphous aggregates with sizes ranging from $100-500 \mathrm{~nm}$ with primary particles of 5-50 nm as reported by the supplier. Other chemicals used include aminopropyl triethoxysilane (APTES) (TCI, 96\%), 1-ethyl-3-(3-dimethylaminopropyl) carbodiimide (EDC) (Chem Impex Int'1, 99.8\%), 2-morpholinoethanesulfonic acid (MES) (Chem Impex Int'1, 99.8\%), $N$-hydroxysuccinimide (NHS) (Chem Impex Int'1, 99.5\%), Fluorescein sodium salt (Fluka), bromooctane (Alfa Aesar, 98\%), bromodecane (Frontier Scientific, 98\%), bromododecane (Alfa Aesar, 98\%), bromohexadecane (TCI, 96\%), brominated vegetable oil (Spectrum), Triton X-100 (TX) (Alfa

88 Aesar), sodium dodecyl sulfate (SDS) (Sigma Aldrich, 99\%), cetyltrimethylammonium bromide (CTAB) (Sigma Aldrich). All chemicals were used as received without further purification.

Preparation of oil-in-water emulsions. Silica particles were first dispersed in oil using a probe sonicator (QSonica Q700). These dispersions were immediately used to fabricate droplets.

92 Unless specified otherwise, droplets were made with a volume ratio of 1:10 oil to surfactant 
solution and were emulsified using a Vortex Genie 2 at its maximum setting (3200 RPM) for 3-5 seconds in 1-dram glass vials. Given that bulk emulsification was used, the droplets had a resultant dispersity in size as well as particle concentration in each droplet.

Brightfield and fluorescence microscopy. To visualize the droplets, $0.5 \mu \mathrm{L}$ of droplets were pipetted into a glass-bottom dish containing surfactant solution. Droplets were gently agitated to disperse them randomly within the dish. Videos of the droplet motion were taken using a Nikon Ti-U inverted microscope and an Andor Zyla 4.2P camera. Typically, several videos for each set of sample conditions were taken and analyzed to provide sufficient statistical data on droplet speeds and particle surface coverages. Fluorescence images were collected with excitation (AT480/30x) and emission (AT535/40m) filters.

Analysis of droplet speed: The videos for speed analysis were taken with a $6 \mathrm{x}$ magnification, 1024 x 1024 resolution, and $30 \mathrm{fps}$ using an Andor Zyla 4.2P camera. Instantaneous droplet speed was analyzed with a MATLAB program as reported previously ${ }^{8,15}$. An average speed was assigned to a sample by averaging the maximum speeds of all the droplets captured within a video. We report the average of the maximum speed and the standard deviation of the maximum speed as seen in Figure 1 and Figure 5. For the data presented in Figure 3, each data point represents the maximum speed attained for a single droplet while it was imaged such that we could correlate that speed directly with a particle surface coverage value. Please note that we did not attempt to account for drift velocity due to convection in any of our reported speed measurements. In our experience, drift velocity is less than $10 \mu \mathrm{m} / \mathrm{s}$ which is much slower than the self-propelled droplets that can move upwards of $200 \mu \mathrm{m} / \mathrm{s}$.

Particle surface functionalization with fluorescein to create fluorescently labeled particles. Functionalization of S13 with APTES. In a $25 \mathrm{~mL}$ round bottom flask, $250 \mathrm{mg}$ of dry S13 particles were dispersed in $10 \mathrm{~mL}$ of acetone. $100 \mu \mathrm{L}$ of $30 \mathrm{wt} \%$ ammonium hydroxide in water was then added, followed by $300 \mu \mathrm{L}$ of APTES. The flask was then sealed with a septum and sonicated in a bath sonicator (Branson 1510) for 1 hour. The solution was then diluted with acetone to a volume of $30 \mathrm{~mL}$ and centrifuged at 7,100 RCF for 10 minutes. The supernatant was decanted and the particles were re-dispersed in acetone through sonication and centrifuged again at 7,100 RCF for 10 minutes to pellet the particles. The supernatant was discarded, and the nanoparticle pellet was collected and dried overnight. The particles were redispersed in $25 \mathrm{~mL}$ of MES buffer ( $0.5 \mathrm{M}, \mathrm{pH} 5)$ for use in the following step. Carbodiimide coupling of fluorescein and amine terminated S13.125 mL of MES buffer (0.5 M, pH 5) was added to a $250 \mathrm{~mL}$ flask, followed by $342.1 \mathrm{mg}$ of fluorescein sodium salt, $165.9 \mathrm{mg}$ of EDC, and $246 \mathrm{mg}$ of NHS. The solution was stirred at room temperature for 30 minutes, and then the $25 \mathrm{~mL}$ MES solution of aminefunctionalized S13 particles was added. The flask was sealed using a septum, covered in foil, and left to react at room temperature for 24 hours while stirring. The solution was then diluted with acetone and excess reagents were removed similarly to the previous step by using centrifugation, washing, and drying. The particles were dispersed in $10 \mathrm{~mL}$ of hexane for use in the following step. Making florescent particles hydrophobic. The $10 \mathrm{~mL}$ of fluorescein-functionalized particles were added to a $25 \mathrm{~mL}$ round bottom flask followed by $200 \mu \mathrm{L}$ of diethylamine and $1.5 \mathrm{~mL}$ of hexadecyltriethoxysilane. The flask was sealed with a septum and left to react for 24 hours while stirring and covered with foil. The solution was then diluted with acetone and excess reagents were removed similarly to the previous steps by using centrifugation, washing, and drying, ultimately producing hydrophobic, fluorescent fumed silica.

Thermogravimetric analysis (TGA) of dry, functionalized silica particles. The weight loss of functionalized silica particles was monitored by TGA (Discovery Series TGA Q5500) ${ }^{16,17}$. TGA 
traces taken for particles produced after each functionalization step are shown in Figure S1. The silica particles were heated in air from $25^{\circ} \mathrm{C}$ to $120^{\circ} \mathrm{C}$ at $10^{\circ} \mathrm{C} / \mathrm{min}$ and held in isotherm for 10 minutes to remove residual solvent. Particles are then heated to $800{ }^{\circ} \mathrm{C}$ at $20{ }^{\circ} \mathrm{C} / \mathrm{min}$. The normalized weight loss was then calculated from mass change between $120^{\circ} \mathrm{C}$ and $800{ }^{\circ} \mathrm{C}$.

Analysis of particle surface coverage on droplets: Surface coverage of droplets coated with fluorescent silica nanoparticles were determined from fluorescence images of the droplets. We approximated the particle coverage as a symmetric, spherical cap of particles. From the images, droplet radius and cap radius are measured to calculate the approximate surface coverage of droplets, as shown in Figure 2. There is notable error inherent within this approximation, given that the particles are not distributed evenly throughout the cap or cap edges, the surface aggregates can become rough or non-spherical due to particle packing, and some distortion in the images is created due to droplet motion during the image exposure, which was necessarily long (30-60 ms) to capture sufficient fluorescence intensity.

\section{Results and Discussion}

To test whether particles at droplet interfaces might influence self-propulsion of solubilizing oil droplets, we began by examining the effect of partially hydrophobic silica particles on the swimming speeds of 1-bromodecane droplets in aqueous Triton X-100 (hereafter, TX). TX is a nonionic surfactant which has previously been shown to generate active oil droplets via micelle-mediated solubilization ${ }^{8}$. Bromodecane was chosen because it is an oil with low water solubility, such that solubilization is expected to be micelle-mediated ${ }^{8}$, and it is denser than water lending to ease of experimentation. We chose partially hydrophobized fumed silica particles (H13L produced by Wacker Chemie, $150-500 \mathrm{~nm}, 50 \%$ coverage with dimethylsiloxy groups and $50 \%$ residual surface silanols) to favor particle wetting by both oil and water and enhance interfacial activity; these particles are, however, still preferentially dispersible in the oil phase. We prepared polydisperse bromodecane droplets with and without $0.5 \mathrm{wt} \% \mathrm{H} 13 \mathrm{~L}$ particles in $0.1 \mathrm{wt} \%$ TX by vortex mixing and examined the droplet dynamics using optical microscopy and droplet tracking analysis (refer to Methods Section for details). Droplets were polydisperse but typical diameters of droplets that were analyzed fell in the range of $20-100 \mu \mathrm{m}$. The bromodecane droplets without particles moved slowly, no more than $3 \mu \mathrm{m} / \mathrm{s}$ (Figure 1a, left) which is likely due to background drift resultant from convection in the imaging chamber and which we do not consider to be self-propelled. However, droplets with the silica particles were self-propelled and moved significantly faster, on the order of $50 \mu \mathrm{m} / \mathrm{s}$, rapidly careening through the imaging chamber (Figure 1b, Video S1). Qualitatively, it was evident from these initial experiments that the particles had a notable effect on the droplet dynamics.

In order to quantify the relationship between particle concentration, surfactant concentration, and droplet speed, we conducted a series of experiments with aqueous TX surfactant concentrations between $0.1 \mathrm{wt} \%$ and $1 \mathrm{wt} \%$ and $\mathrm{H} 13 \mathrm{~L}$ particle concentrations in bromodecane between $0 \mathrm{wt} \%$ and $2 \mathrm{wt} \%$. For each sample, we used a standardized procedure in which a small number of droplets (typically less than 20 droplets in $0.5 \mu \mathrm{L}$ of solution) were extracted from the emulsion sample vial and added to a glass-bottom dish containing $1 \mathrm{~mL}$ the same surfactant concentration in which the droplets were prepared. The solution was gently agitated to randomly disperse the droplets, and videos of the droplets were collected over 60 seconds. Droplet trajectories and instantaneous speeds were analyzed using Matlab image analysis ${ }^{8,15}$. Given that the droplets often swam in curved trajectories and exhibited variation in instantaneous speed that was dependent on the path, we used the maximum speed each droplet reached during the video as 
the basis for comparison. Averages and standard deviations for the resultant maximum speeds for each set of experimental conditions are given in Figure 1b and Table S1. Droplets exhibited faster speeds in higher surfactant concentrations for a given particle concentration. These trends with surfactant concentration are consistent with a solubilization-driven, micelle-mediated propulsion mechanism $^{2}$. Higher particle concentration, however, did not always correlate to faster speeds, and instead there was a maximum in droplet speed at intermediate particle concentrations (Figure 1b). The particle concentration yielding the fastest speeds varied slightly as a function of TX concentration with the droplet speed peaking at around $0.2 \mathrm{wt} \%$ to $0.5 \mathrm{wt} \% \mathrm{H} 13 \mathrm{~L}$ particles. At lower particle concentrations, we could not see the particles clearly within the droplets, but at higher particle concentrations, we observed large irregular particle aggregates on the droplets' surfaces (Figure 1b inset). We suspected that the particle concentration in the droplet was correlated with the number of particles at the droplet interface and the total displaced oil-water interfacial area, which was, in turn, affecting the droplet speeds.

To directly correlate the particle concentrations to droplet interfacial coverage and speeds, we needed to be able to directly visualize the particles at the droplet surface, such as with fluorescence. We aimed to modify the fumed silica particles with a fluorescent dye, fluorescein, while still retaining a particle surface activity similar to that of the H13L (Figure 2a). Starting from pristine hydrophilic fumed silica, we functionalized the silanol surface with aminopropyl triethoxysilane (APTES) and then coupled the surface amine with the carboxylic acid of fluorescein sodium salt via carbodiimide coupling chemistry using 1-ethyl-3-(3dimethylaminopropyl) carbodiimide (EDC) and $N$-hydroxysuccinimide (NHS). At this stage, the particles were still hydrophilic and dispersed easily in water, so we further functionalized remaining surface silanol groups with hexadecyltrimethoxysilane to render the particles more hydrophobic. After functionalization, the particles were fluorescent and dispersible in the bromodecane. Please refer to the Methods section for specific reaction details and particle surface analysis.

To test if the hydrophobized fluorescent silica could be used for particle visualization, the particles were dispersed into bromodecane at $2 \mathrm{wt} \%$ and emulsions were prepared by vortex mixing the bromodecane/particle mixture in $0.5 \mathrm{wt} \% \mathrm{TX}$. Immediately upon placing the droplets into fresh surfactant solution for imaging, the droplets were non-active and most of the fluorescent particles were dispersed inside the droplets. Over a short time, particles that were circulating inside the droplets began to accumulate at the droplet surface and aggregate, eventually being pushed towards the rear pole of the droplet as the droplet started to propel quickly forward (Figure 2b, Video S2). Using the fluorescent particles, we could visualize the degree of droplet interfacial coverage once the droplets reached a steady-state speed, which we approximated from the micrographs as the surface area of a spherical cap (Figure $\mathbf{2 b}$ ). This is a rough estimate, as the particles were not perfectly packed at the interface, the edges of the particle cap were not straight, and we could not visualize the 3D surface coverage around all sides of the droplet simultaneously. There was also some image blur in the fluorescence micrographs because the droplets were moving during the camera exposure time, which was necessarily long $(30-60 \mathrm{~ms})$ in order to capture sufficient light intensity to visualize the fluorescent particles.

Despite the approximations necessary to quantify surface coverage, we could still visualize the particles sufficiently to correlate the particle coverage with droplet behavior. Bromodecane droplets with fluorescent particle concentrations in the range of $0.2-2 \mathrm{wt} \%$ were prepared in 0.5 $\mathrm{wt} \% \mathrm{TX}$ and videos of the droplets under both brightfield and fluorescence were collected (see Video S3 as an example of a fluorescence video). From these videos, both speed and surface 
coverage were measured for individual droplets; surface coverages were converted to cap coverage angle, $\theta_{c}$, where $\theta_{c}=0^{\circ}$ is an uncovered droplet and $\theta_{c}=180^{\circ}$ is a fully covered droplet. The speed of the droplets as a function of the coverage angle and particle concentration is shown in Figure 3a. Droplet speed showed a non-monotonic dependence on particle coverage with the propulsion speed lowest at small $\left(\theta_{c} \approx 0^{\circ}\right)$ and large $\left(\theta_{c} \approx 180^{\circ}\right)$ surface coverages. The maximum speed attained was in the range of about $300 \mu \mathrm{m} / \mathrm{s}$ at approximately $40 \%$ surface coverage, with $\theta_{c}$ just below $90^{\circ}$. Particle concentrations correlated roughly with surface coverage, as expected based on the data in Figure 1b, although there was still notable variation likely due to dispersity in the numbers of particles within each droplet resultant from the preparation method. The fact that there exists significant variation in surface coverage as a function of initial particle concentration in the bromodecane also explains the relatively large error bars of Figure 1b.

The evolution of droplet speed as a single droplet solubilizes over time in $0.5 \mathrm{wt} \% \mathrm{TX}$ is shown in Figure 3b. With moderate starting surface coverage $\left(\theta_{c} \approx 50^{\circ}\right)$, the droplet initially propelled at $\sim 250 \mu \mathrm{m} / \mathrm{s}$ and maintained a similar speed for about 30 minutes, at which point the speed began to decrease until eventually the droplet was nonactive and completely coated with particles. The surface area of the initial particle patch was estimated to be approximately 11,600 $\mu m^{2}$ and the surface area of the final, fully-covered droplet was estimated to be approximately $10,200 \mu \mathrm{m}^{2}$; this indicates that the vast majority of particles are irreversibly adsorbed such that as the droplet volume shrinks, the total surface coverage by particles stays constant and the percent surface coverage increases. A small decrease in particle-covered surface area might be attributed to adsorbed particles jamming more tightly. Using the trajectory from Figure $\mathbf{3 b}$, we estimate that this droplet had a cruising range of about 0.8 meter over its lifetime of an hour. Droplets with different starting surface coverages or droplet volumes would have different cruising ranges.

We consider the following framework to conceptually rationalize the trends observed in the data of Figure 3. The measured propulsion speed is around $300 \mu \mathrm{m} / \mathrm{s}$ for a half-coated droplet, which, upon using a droplet radius $a_{d r o p}=50 \mu \mathrm{m}$ and kinematic viscosity of $1 \frac{\mu \mathrm{m}^{2}}{\mathrm{~s}}$, gives a Reynolds number $R e=1.5 \times 10^{-2}$. Thus, the droplet motion is in the creeping flow, or low Reynolds number, regime. The oil undergoes solubilization into the aqueous solution, through the formation of oil-filled micelles that are stabilized via uptake of surfactant monomer adsorbed at the oil-water interface. We assume that the flux $j_{m}$ of oil filled micelles into the aqueous solution is constant. A mass balance on the oil in the drop shows that $j_{m}=-\left(\frac{1}{V_{m}}\right) \frac{d a_{d r o p}}{d t}$, where $a_{d r o p}$ is the radius of the oil drop, $V_{m}$ is the volume of an oil filled micelle, and $t$ is time. The rate of change of the drop radius is small, $O(0.01 \mu \mathrm{m} / \mathrm{s})$, compared to the propulsion velocity observed in experiments, $O(100 \mu \mathrm{m} / \mathrm{s}))$; hence, the drop radius is assumed to be essentially constant during propulsion. The solubilization consumes adsorbed surfactant at a rate $d j_{m}$, where $d$ is the number of surfactant monomers per oil filled micelle, or aggregation number. Replenishment of surfactant at the oil-water interface occurs via adsorption of monomers from the bulk solution. The bulk concentration of surfactant monomer is expected to remain uniform and equal to the critical micelle concentration $\left(C_{C M C}\right)$ during the solubilization process, as a result of an abundance of empty micelles that, via rapid dissociation, rectify the deficit in bulk surfactant concentration due to adsorption $^{18}$.

The concentration of adsorbed surfactant may vary along the droplet surface via surface diffusion and advection with the local interfacial fluid flow. Such variation will lead to gradients in surface tension along the oil-water interface that, in turn, drive Marangoni stresses, potentially causing droplet propulsion. The droplets propel with the particle-uncoated portion of their surface 
facing forward (Figure 2b, Figure 3). We therefore expect that the adsorbed surfactant concentration is higher at the front of the drop than at the back; the surface tension follows the opposite trend: highest at the back and lowest at the front (Figure 4). Thus, Marangoni stresses drive an interfacial flow toward the back of the droplet (i.e., the interface is "pulled" backward by the higher interfacial tension at the rear of the drop), as depicted in Figure 4. The flow also advects particles rearward. The "thrust" supplied by this Marangoni flow must be balanced by the "drag" on the droplet due to its translation, to ensure that the freely suspended droplet does not experience thrust, with its uncoated portion facing forward.

The relative importance of surface diffusion to advection is characterized by a Péclet number $P e=U a / D_{s}$, where $U$ is the propulsion speed of the drop, and $D_{s}$ is the surface diffusion coefficient. Using a typical speed $U=100 \mu \mathrm{m} / \mathrm{s}$, drop size $a_{d r o p}=50 \mu \mathrm{m}$, and $D_{s}=$ $150 \mu \mathrm{m}^{2} / \mathrm{s}$ yields $P e=33$. (We have assumed, in the absence of better information, that the surface diffusion coefficient of surfactant monomer is equal to the bulk diffusion coefficient ${ }^{19}$.) Hence, advection dominates diffusion. In this regime, it has recently been predicted that droplets in a micellar solution (with bulk surfactant concentration above the $C_{C M C}$ ) can spontaneously selfpropel due to a Marangoni instability ${ }^{18}$, resulting from the nonlinear dependence of the advective interfacial flux of surfactant on the interfacial velocity and surfactant concentration. A similar selfpropulsion mechanism for a solid particle coated by enzymes that are mobilized by diffusiophoretic flows, generated by a concentration gradient in the product species of the enzymatic reaction, has also recently been proposed ${ }^{20}$. Indeed, the particle free $(0 \mathrm{wt} \%)$ bromodecance droplets in Figure 3 do exhibit self-propulsion, which we believe is due to a Marangoni instability.

We thus propose that the non-monotonic dependence on the droplet speed with increasing cap angle $\theta_{c}$ can be understood as follows. First, we assume that oil solubilization is inhibited over the particle-coated portion of the drop, due to the reduction in oil-water interfacial area in this region. Hence, as $\theta_{c}$ is increased between $0 \leq \theta \leq \pi / 2$ the asymmetry in the solubilization over the surface of the droplet increases, reaching a maximum at $\theta_{c}=\pi / 2$. This increase in solubilization asymmetry promotes an increase in the asymmetry of the adsorbed surfactant concentration distribution, which would lead to a greater Marangoni thrust and hence propulsion speed. However, we also expect that Marangoni flows are suppressed, or at least weakened, over the particle-coated portion, again due to the reduction in oil-water interface. Hence, we propose that the particles have dual, competing effects: first, to promote adsorbed surfactant gradients, and hence Marangoni flow, via an increase in solubilization asymmetry; and, second, to decrease the available surface area over which Marangoni flow can occur. Upon increasing $\theta_{c}$ from zero, the former effect outweighs the latter leading to an increase in drop speed up until $\theta_{c} \approx \pi / 2$. Now, $\theta_{c}=\pi / 2$ represents the cap angle that generates the maximum asymmetry in solubilization. Hence, increasing $\theta_{c}$ beyond $\pi / 2$ leads to a decrease in the solubilization asymmetry, which would reduce the propulsion speed, along with a decrease in the oil-water interfacial area available for Marangoni flow. For a fully particle-coated drop we suppose that solubilization is completely inhibited and thus the droplet speed should fall to zero, which is seen in Figure 3.

The above argument proposes a mechanism whereby adsorbed particles can alter the speed of a droplet that would self-propel even in an absence of such particles (Figure 4a). However, we had also previously observed that droplets of bromodecane in $0.1 \mathrm{wt} \% \mathrm{TX}$, which are not selfpropelled in the absence of particles, similarly experience faster swimming speeds in the presence of particles (Figure 1). These droplets do still undergo solubilization, albeit at a slower rate, which 
presumably is uniform over their surface since self-propulsion (i.e. lateral movement at speeds above drift velocity) does not occur in the absence of particles. Evidently, the presence of particles at the droplet surface can lead to self-propulsion of a droplet that otherwise would be non-motile. We suggest that this induced propulsion may occur due to a non-uniform surface coverage of particles, which would break the uniformity of the solubilization via displacement of oil-water interfacial area, as explained in the above paragraph. The non-uniform coverage itself may arise spontaneously as a result of, for example, differential rates of particle adsorption to the interface resultant from particle polydispersity (Figure 4b). A second possible source of spontaneous symmetry breaking for a non-self-propelled droplet could be the presence of an oil-filled micelle gradient generated by another nearby solubilizing droplet ${ }^{8,12}$. Once self-propulsion has been initiated, however, the particles should be advected toward the rear of the droplet by the Marangoni flow, further exacerbating the asymmetry in a positive feedback response and leading to the eventual formation of the particle cap (as seen in Video S2).

To explore the generality of this particle-assisted propulsion mechanism, we examined the swimming behaviors of bromodecane in several different surfactants and surfactant concentrations. Bromodecane droplets containing $1 \mathrm{wt} \%$ fluorescent particles were emulsified in anionic sodium dodecyl sulfate (SDS), cationic cetyl trimethyl ammonium bromide (CTAB), and nonionic TX of varying concentration. Individual droplets within each sample were tracked and characterized to determine their maximum speed and particle coverage. Only droplets that had between 30\% and 50\% surface coverage were included in the data shown in Figure 5 and Table S2-S3 to account for possible differences in particle surface activity under the varying surfactant conditions $^{21}$. Without particles, bromodecane droplets had slow swimming speeds in all concentrations of the ionic surfactants, SDS and CTAB, $(<20 \mu \mathrm{m} / \mathrm{s})$, although the bromodecane was solubilizing at a noticeable rate $(0.03$ and $0.06 \mu \mathrm{m} / \mathrm{min}$ respectively, Table S4). Inclusion of particles increased the droplet speed by over an order of magnitude for many conditions (Video S4), and the enhancement increased at higher SDS and CTAB concentrations. Addition of $0.25 \mathrm{M}$ $\mathrm{NaCl}$ to $1 \mathrm{wt} \%$ and $5 \mathrm{wt} \%$ SDS or $1 \mathrm{wt} \%$ and $2.5 \mathrm{wt} \%$ CTAB marginally increased droplet speeds without particles and also increased solubilization rates slightly, potentially due to screening of the electrostatic repulsion between the charged micelles and interface (Table S2, S4). Addition of salt in the presence of particles, however, led to significantly faster droplet speeds in $1 \mathrm{wt} \%$ SDSparticles-salt $(345.9 \pm 74.4 \mu \mathrm{m} / \mathrm{s})$ and in $1 \mathrm{wt} \%$ CTAB-particles-salt $(251.6 \pm 42.9 \mu \mathrm{m} / \mathrm{s})$. Adding salt did not significantly influence the speed of droplets in nonionic surfactant TX-stabilized droplets with or without particles. These results suggest that salt affects the propulsion via surfactant-salt interactions or surfactant-particle-salt interactions, rather than just interactions between the salt and particles alone. In the case of SDS and CTAB, addition of salt may also cause a change in the $\mathrm{CMC}^{22,23}$, and salt also may also allow the particles to pack together more tightly providing greater interfacial coverage by screening charge on the fumed silica resulting from ionic surfactant-particle association ${ }^{24}$.

Given that the oil droplets are propelled by interfacial tension gradients resultant from oilsurfactant interactions, we wondered to what extent oil droplets of varying chemistry and solubilization rates would be influenced by particles. We measured the speeds of 1-bromooctane, 1-bromodecane, 1-bromododecane, 1-bromohexadecane, and brominated vegetable oil droplets with and without $1 \mathrm{wt} \%$ fluorescent particles in $0.5 \mathrm{wt} \% \mathrm{TX}$ (Figure 5b, Table S3). Again, we only consider here droplets with particle surface coverage in the range of 30-50\%. All oils except the brominated vegetable oil exhibited enhanced propulsion speeds with particles present. Without particles, the solubilization rates of these oils are $0.41,0.24,0.13,<0.01$, and $<0.01 \mu \mathrm{m} / \mathrm{min}$, 
respectively, where the bromohexadecane and brominated vegetable oil did not solubilize to a measurable extent within one hour (Table S4). We do believe, however, that the bromohexadecane still solubilizes, albeit at a slow rate, given previous reports of measurable solubilization of hexadecane in $\mathrm{TX}^{25}$. Brominated vegetable oil is quite viscous containing molecules of even higher molecular weight and is thus expected to solubilize even more slowly than the bromohexadecane. This observation is consistent with the idea that solubilization and the generation of oil gradients is a requirement for propulsion; while particles can significantly enhance the speed of droplets that undergo some degree of solubilization, particles alone cannot generate propulsion in the absence of solubilization. Rather, particles can only lead to an asymmetry in solubilization for a droplet that would solubilize in the absence of particles.

\section{Conclusion}

In summary, we have demonstrated that adsorption of silica nanoparticles at the interface of a solubilizing oil droplet in surfactant solution can significantly accelerate the droplets' selfpropulsion speed. The polarization of the particles across the droplet surface arises spontaneously to form a cap, and using fluorescent particle visualization, we correlated the degree of particle surface coverage on bromodecane droplets to the droplet speed in TX surfactant. Slowest speeds were found at the lowest and highest surface coverages and the fastest speeds were achieved at intermediate surface coverages of about $40 \%$. The particle-assisted propulsion acceleration was further demonstrated in nonionic, anionic, and cationic surfactants and a range of oils with varying solubilization rates. We propose a possible mechanism for propulsion enhancement in which particles at the droplet interface hinder solubilization by displacing oil-water interfacial area, providing asymmetry in the distribution of oil-filled micelles and adsorbed surfactant along the droplet surface. The latter has the consequence of increasing variations in interfacial tension at the drop surface hence the Marangoni thrust for self-propulsion. However, particles may also decrease the available surface area over which Marangoni flow can occur, leading to competing effects as a function of particle surface coverage. Future work will include development of fluid mechanical models to test the proposed mechanistic hypotheses. Approaches by which to modulate the distribution of solubilization across droplet interfaces, such as by addition of particles, may provide a facile route to tuning active colloid speeds and dynamics. Further exploration involving stimuliresponsive particles ${ }^{26}$ or droplets containing multiple oils ${ }^{27}$ with particles at droplet-internal oil-oil interfaces ${ }^{28}$ may provide new opportunities for tuning the behaviors of swimming droplets.

Acknowledgments. We gratefully acknowledge financial support from the American Chemical Society Petroleum Research Fund (Grant 59833-DNI10), the Army Research Office (Grant W911NF-18-1-0414), and from the Charles E. Kaufman Foundation of the Pittsburgh Foundation. We thank Pepijn Moerman for the Matlab code which we used in tracking droplet speeds. 
a droplets without particles

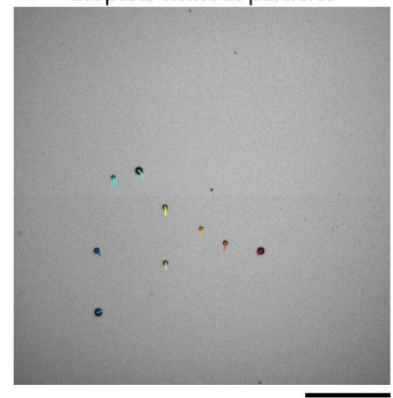

droplets with $0.5 \mathrm{wt} \%$ particles

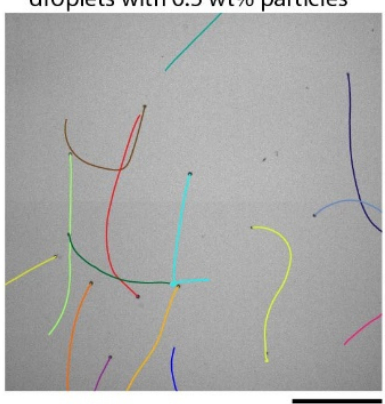

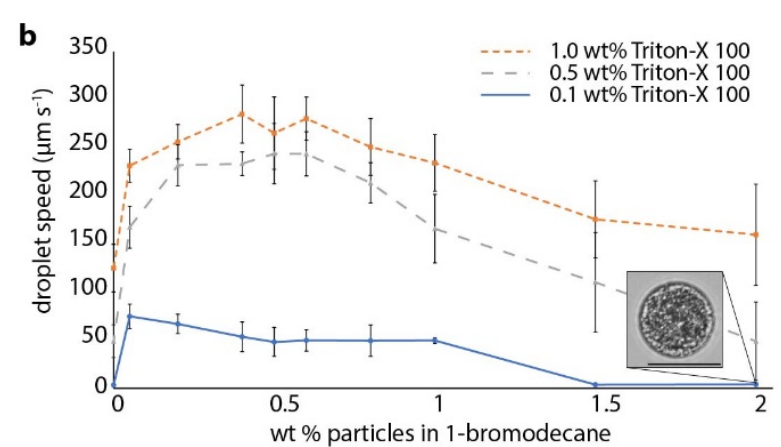

Figure 1. Silica particles affect the self-propulsion of oil droplets in surfactant solution. a. 1Bromodecane oil droplets without particles and with $0.5 \mathrm{wt} \% \mathrm{H} 13 \mathrm{~L}$ silica were dispersed in 0.1 $\mathrm{wt} \% \mathrm{TX}$ and the droplet trajectories were analyzed. Shown are the droplet trajectories over a 60 second period. Droplets without particles were not active, and droplets with particles swam much faster, reaching peak speeds of about $50 \mu \mathrm{m} / \mathrm{s}$. Scale, $1 \mathrm{~mm}$. b. The speed of bromodecane droplets was investigated for various aqueous TX concentrations and H13L particle concentrations. The speed of a droplet was defined as the maximum speed reached during the droplet's lifetime in the video frame. Data shown represent the average and standard deviation for a sample size of at least 10 droplets per experimental condition. The inset shows an optical micrograph of a droplet at 2 $\mathrm{wt} \%$ particle concentration in $0.1 \mathrm{wt} \% \mathrm{TX}$ where the packing of the nanoscale particles at the droplet surface become visible, showing high surface coverage. When higher concentrations of TX surfactant are used, we do not often see such fully packed surfaces and the droplets may experience significant motion even at $2 \mathrm{wt} \%$ particles. Scale, $50 \mu \mathrm{m}$.
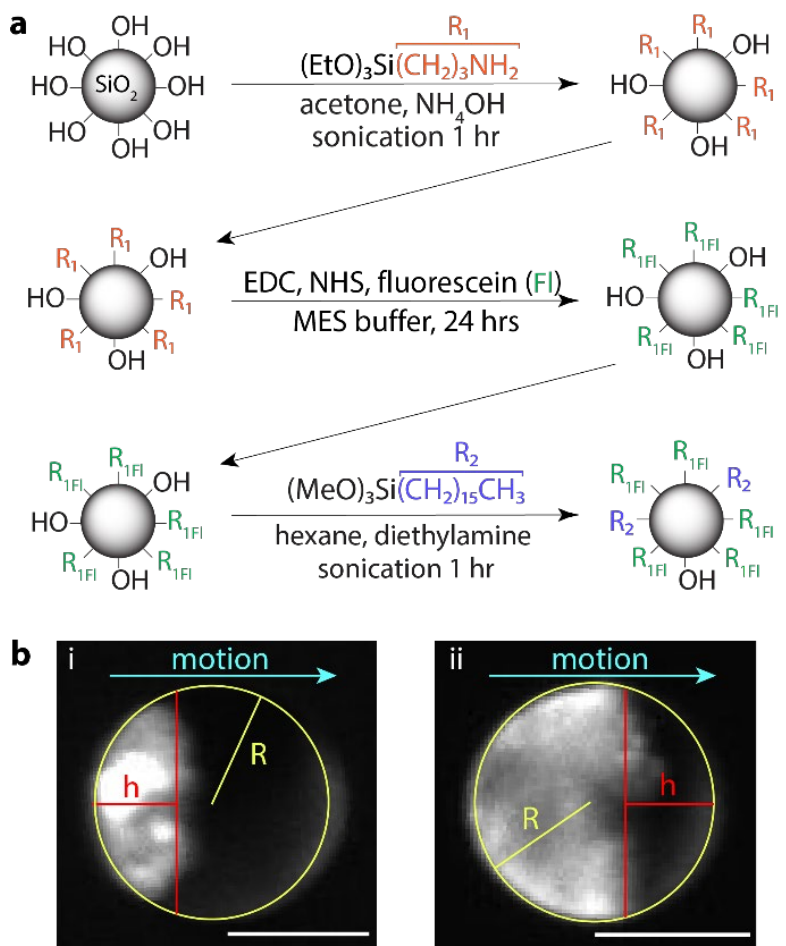

Figure 2. Fluorescence visualization of particles at droplet interfaces. a. Schematic describing the preparation of fluorescent, hydrophobic fumed silica. b. The particle surface coverage of a 
droplet was estimated using fluorescence micrographs. Fluorescence images of two different droplets, both prepared with $1 \mathrm{wt} \%$ fluorescent particles in bromodecane with $0.5 \mathrm{wt} \% \mathrm{TX}$, are shown as examples. Overlaid diagrams illustrate how particle surface coverage was estimated by assuming a spherical cap, where $h$ is the height of the cap and $R$ is the radius of the droplet (and cap). For (i), estimated fractional surface coverage $=h / 2 R=32 \%$, and for (ii) fractional surface coverage $=1-h / 2 R=70 \%$. Scale, $50 \mu \mathrm{m}$.
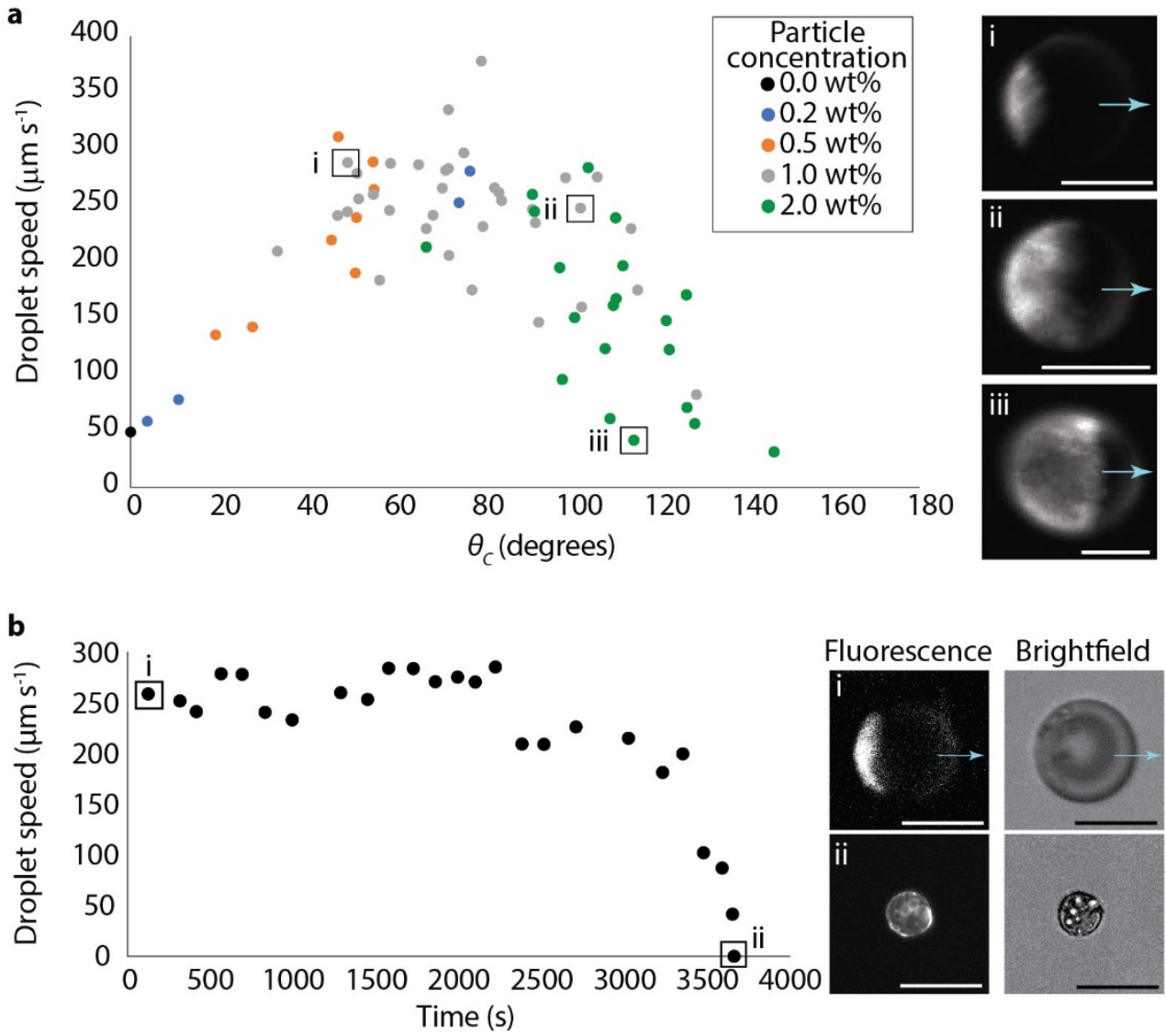

Figure 3. Degree of droplet surface covered by particles affects droplet speed. The scatterplot shows the relationship between bromodecane droplet speed and surface coverage with particles in $0.5 \mathrm{wt} \% \mathrm{TX}$. Surface coverage is plotted as coverage cap angle $\theta_{c}$, where $\theta_{c}=0^{\circ}$ corresponds to no particle coverage and $\theta_{c}=180^{\circ}$ corresponds to complete coverage. Each datapoint represents a single droplet's surface coverage and highest speed reached during imaging. The color coding of the datapoints represents the initial concentration of particles used when preparing the droplet. Black $=0.0 \mathrm{wt} \%$, blue $=0.2 \mathrm{wt} \%$, orange $=0.5 \mathrm{wt} \%$, grey $=1.0 \mathrm{wt} \%$, and green $=2.0 \mathrm{wt} \%$ fluorescent particles in bromodecane. The initial particle concentration showed a general correlation with droplet surface coverage, where higher particle concentrations were more likely to produce droplets with higher surface coverages. Fluorescence micrographs of three exemplary droplets are shown and the arrows represent direction of droplet motion. Scale, $50 \mu \mathrm{m}$. b. The scatterplot shows the evolution of a single bromodecane droplet's speed over its lifetime of about an hour in $0.5 \mathrm{wt} \% \mathrm{TX}$. The droplet started with fast speeds and moderate particle coverage $\left(\theta_{c} \approx\right.$ $50^{\circ}$ ). Over time, the particle coverage increased while the droplet volume decreased due to 
solubilization, until eventually the droplet motion ceased with $\theta_{c} \approx 180^{\circ}$. Fluorescence and brightfield micrographs are given at right. The arrows represent the direction of droplet motion. Scale, $100 \mu \mathrm{m}$.
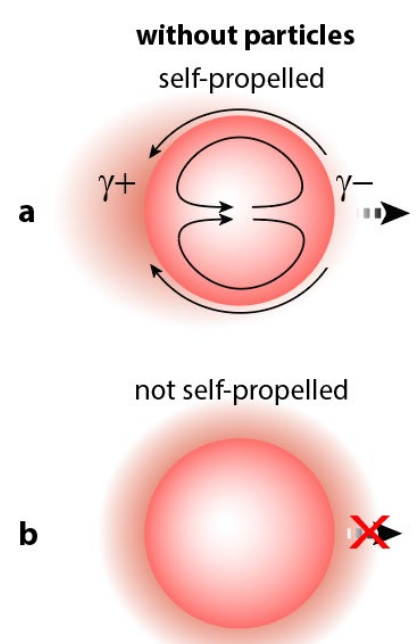

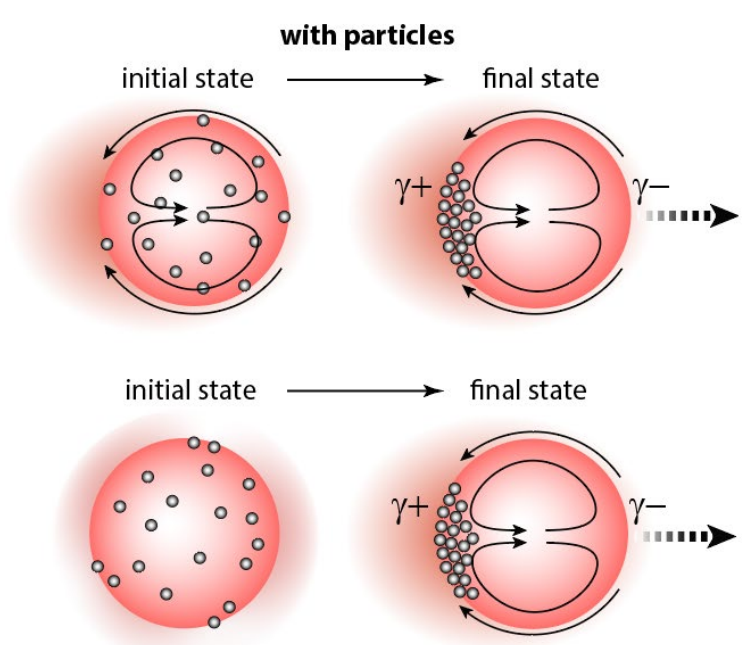

Figure 4. Spontaneous polarization of particles on solubilizing oil droplets leads to enhanced self-propulsion. Schematic representations for how particle polarization may arise within (a) a droplet that already exhibits self-propulsion in the absence of particles and (b) a droplet that solubilizes but which does not exhibit self-propulsion. $\gamma+$ indicates an elevated interfacial tension and $\gamma$ - indicates a lowered interfacial tension such that Marangoni flow proceeds from the right to left along the droplet surface (as indicated by the arrows) propelling the droplet rightward. a. An oil droplet that solubilizes and self-propels will advect particles along the oil-water interface to the droplet rear, leading to the formation of the particle cap which further affects droplet propulsion speed. b. A solubilizing droplet that does not exhibit self-propulsion has a presumed symmetrical gradient of solubilized oil as depicted (red shading). When particles are introduced, spontaneous asymmetry in the solubilized oil gradient may arise due to inhomogeneous distribution of particles adsorbed to the oil-water interface. The asymmetry in the solubilized oil gradient will generate local fluctuations in in particle aggregation at the oil-water interface that over time feeds back into the formation of the particle cap, leading to self-propulsion. 


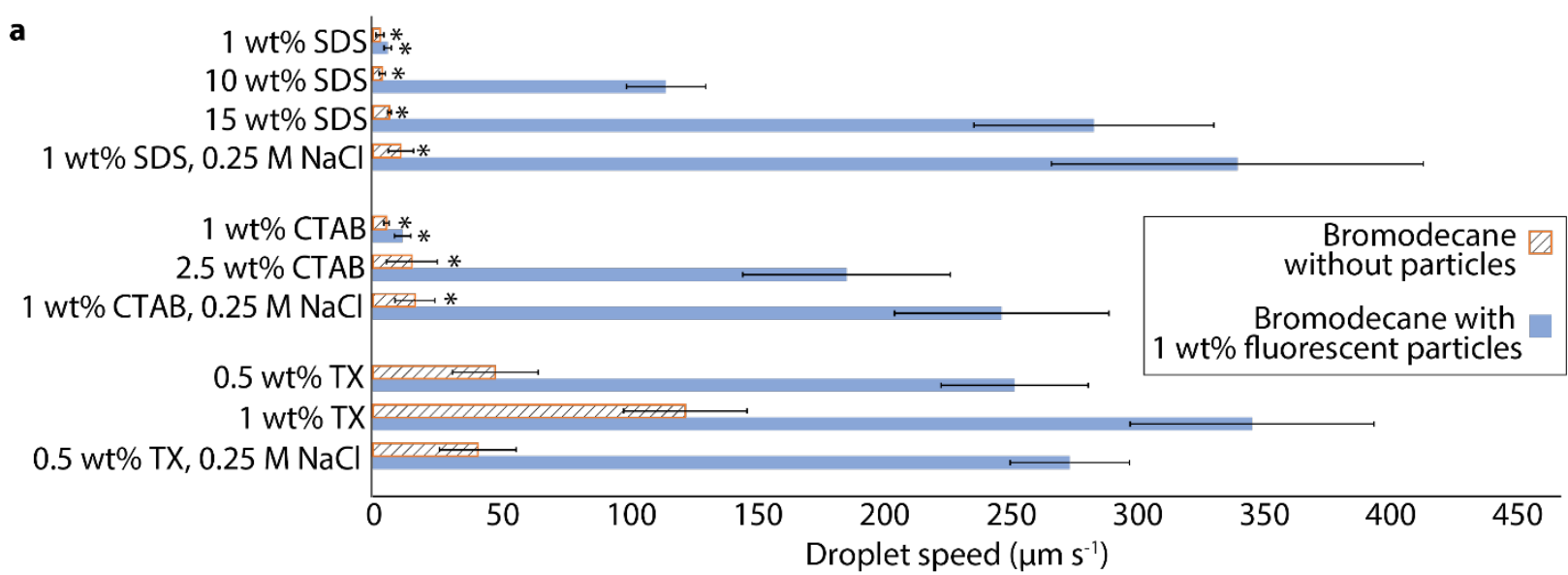

b

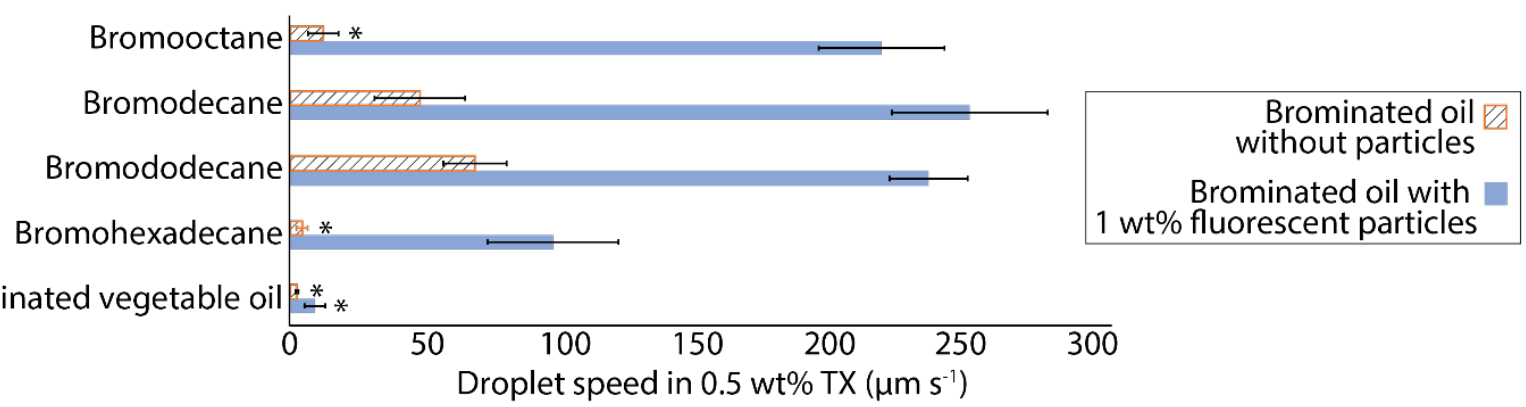

Figure 5. Enhanced self-propulsion of oil droplets of varying oil and surfactant chemistry. a. Speeds of bromodecane droplets with and without $1 \mathrm{wt} \%$ fluorescent silica particles were measured in different concentrations of SDS, CTAB, and TX surfactant, with and without $\mathrm{NaCl}$. The asterisk $(*)$ indicates that the droplets were noticeably drifting rather than self-propelling, as distinguished by the droplets all moving in the same direction. The speeds of droplets prepared with ionic surfactants, SDS and CTAB, were sensitive to the addition of $0.25 \mathrm{M} \mathrm{NaCl}$, whereas droplets in nonionic TX were not. b. Speeds of various brominated oils with and without $1 \mathrm{wt} \%$ fluorescent silica particles were measured in $0.5 \mathrm{wt} \%$ TX. See Table $\mathbf{S} 4$ for solubilization rates. All oils, except for brominated vegetable oil which had indetectable solubilization, showed significant enhancement in self-propulsion speed due to the surface adsorption of silica particles. Each bar shows the average and standard deviation of a minimum of 5 droplet measurements. The data plotted in (a, b) is tabulated in Table S2, S3. Only droplets with 30-50\% surface coverage of particles were included in this data to account for possible differences in particle surface adsorption with variation in oil or surfactant.

\section{References}

1. Dey, K. K. \& Sen, A. Chemically Propelled Molecules and Machines. J. Am. Chem. Soc. 139, 7666-7676 (2017).

2. Maass, C. C., Krüger, C., Herminghaus, S. \& Bahr, C. Swimming Droplets. Annu. Rev. Condens. Matter Phys. 7, 171-193 (2016).

3. Pourrahimi, A. M. \& Pumera, M. Multifunctional and Self-propelled Spherical Janus Nano/micromotors: Recent Advances. Nanoscale 10, 16398-16415 (2018).

4. Lach, S., Yoon, S. M. \& Grzybowski, B. A. Tactic, Reactive, and Functional Droplets Outside of Equilibrium. Chem. Soc. Rev. 45, 4766-4796 (2016). 
5. Toyota, T., Maru, N., Hanczyc, M. M., Ikegami, T. \& Sugawara, T. Self-propelled Oil Droplets Consuming 'Fuel' Surfactant. J. Am. Chem. Soc. 131, 5012-5013 (2009).

6. Hanczyc, M. M., Toyota, T., Ikegami, T., Packard, N. \& Sugawara, T. Fatty Acid Chemistry at the Oil-water Interface: Self-propelled Oil Droplets. J. Am. Chem. Soc. 129, 9386-9391 (2007).

7. Izri, Z., Van Der Linden, M. N., Michelin, S. \& Dauchot, O. Self-propulsion of Pure Water Droplets by Spontaneous Marangoni-stress-Driven Motion. Phys. Rev. Lett. 113, 15 (2014).

8. Meredith, C. H. et al. Predator-prey Interactions Between Droplets Driven By Nonreciprocal Oil Exchange. Nat. Chem. 12, 1136-1142 (2020).

9. Jin, C., Kru-ger, C. \& Maass, C. C. Chemotaxis And Autochemotaxis of Self-propelling Droplet Swimmers. Proc. Natl. Acad. Sci. U. S. A. 114, 5089-5094 (2017).

10. Michelin, S., Lauga, E. \& Bartolo, D. Spontaneous Autophoretic Motion of Isotropic Particles. Phys. Fluids 25, (2013).

11. Lagzi, I., Soh, S., Wesson, P. J., Browne, K. P. \& Grzybowski, B. A. Maze Solving By Chemotactic Droplets. J. Am. Chem. Soc. 132, 1198-1199 (2010).

12. Moerman, P. G. et al. Solute-mediated Interactions Between Active Droplets. Phys. Rev. E 96, 032607 (2017).

13. Binks, B. P. \& Horozov, T. S. Colloidal Particles At Liquid Interfaces. Cambridge University Press (2006).

14. Binks, B. P. Colloidal Particles At A Range Of Fluid-Fluid Interfaces. Langmuir 33, 6947-6963 (2017).

15. Crocker, J. C. \& Grier, D. G. Methods Of Digital Video Microscopy For Colloidal Studies. J. Colloid Interface Sci. 179, 298-310 (1996).

16. Wisser, F. M. et al. Detection Of Surface Silanol Groups On Pristine And Functionalized Silica Mixed Oxides And Zirconia. J. Colloid Interface Sci. 374, 77-82 (2012).

17. Mueller, R., Kammler, H. K., Wegner, K. \& Pratsinis, S. E. OH Surface Density Of SiO2 And TiO2 By Thermogravimetric Analysis. Langmuir 19, 160-165 (2003).

18. Morozov, M. Adsorption Inhibition By Swollen Micelles May Cause Multistability In Active Droplets. Soft Matter 16, 5624-5632 (2020).

19. Fang, X. W., Zhao, S., Mao, S. Z., Yu, J. Y. \& Du, Y. R. Mixed micelles of cationicnonionic surfactants: NMR self-diffusion studies of Triton X-100 and cetyltrimethylammonium bromide in aqueous solution. Colloid Polym. Sci. 281, 455-460 (2003).

20. De Corato, M., Pagonabarraga, I., Abdelmohsen, L. K. E. A., Sánchez, S. \& Arroyo, M. Spontaneous Polarization and Locomotion of an Active Particle with Surface-mobile Enzymes. Phys. Rev. Fluids 5, 1-11 (2020).

21. Katepalli, H. \& Bose, A. Response of Surfactant Stabilized Oil-in-Water Emulsions to the Addition of Particles in an Aqueous Suspension. Langmuir 30, 12736-12742 (2014).

22. Gurkov, T. D. et al. Ionic Surfactants On Fluid Interfaces: Determination Of The Adsorption; Role Of The Salt And The Type Of The Hydrophobic Phase. Colloids Surfaces A Physicochem. Eng. Asp. 261, 29-38 (2005).

23. Mahbub, S. et al. Conductometric And Molecular Dynamics Studies Of The Aggregation Behavior Of Sodium Dodecyl Sulfate (SDS) And Cetyltrimethylammonium Bromide (CTAB) In Aqueous And Electrolytes Solution. J. Mol. Liq. 283, 263-275 (2019).

24. Wang, H., Singh, V. \& Behrens, S. H. Image Charge Effects On The Formation Of 
Pickering Emulsions. J. Phys. Chem. Lett. 3, 2986-2990 (2012).

540 25. Zhong, H. et al. Aggregate-based sub-CMC Solubilization of Hexadecane by Surfactants.

$541 \quad$ RSC Adv. 5, 78142-78149 (2015).

542 26. Tang, J., Quinlan, P. J. \& Tam, K. C. Stimuli-responsive Pickering Emulsions: Recent

$543 \quad$ Advances And Potential Applications. Soft Matter 11, 3512-3529 (2015).

544 27. Balaj, R. V. \& Zarzar, L. D. Reconfigurable Complex Emulsions: Design, Properties, And $545 \quad$ Applications. Chem. Phys. Rev. 1, 011301 (2020).

546 28. Cheon, S. I., Batista Capaverde Silva, L., Ditzler, R. \& Zarzar, L. D. Particle Stabilization of Oil-Fluorocarbon Interfaces and Effects on Multiphase Oil-in-Water Complex 


\section{Supporting information for:}

\section{Interfacially-adsorbed particles enhance the self-propulsion of oil droplets in aqueous surfactant}

Seong Ik Cheon ${ }^{1}$, Leonardo Batista Capaverde Silva ${ }^{2}$, Aditya Khair ${ }^{3}$, Lauren D. Zarzar*1,2,4

1. Department of Chemistry, The Pennsylvania State University, University Park, PA 16802

2. Department of Materials Science and Engineering, The Pennsylvania State University, University Park, PA 16802

3. Department of Chemical Engineering, Carnegie Mellon University, Pittsburgh, PA 15213

4. Materials Research Institute, The Pennsylvania State University, University Park, PA, 16802

*Correspondence to 1dz4@psu.edu

Figure S1. Thermogravimetric analysis traces of particles produced during the different steps of fluorescent particle synthesis. The silica particles were heated in air from $25{ }^{\circ} \mathrm{C}$ to $120{ }^{\circ} \mathrm{C}$ at $10{ }^{\circ} \mathrm{C} / \mathrm{min}$ and held in isotherm for 10 minutes to remove residual solvent. Particles are then heated to $800{ }^{\circ} \mathrm{C}$ at $20^{\circ} \mathrm{C} / \mathrm{min}$. The normalized weight loss was then calculated from mass change between $120^{\circ} \mathrm{C}$ and $800^{\circ} \mathrm{C}$. a, Analysis of amine-terminated S13 silica, functionalized with aminopropyl triethoxysilane. b, Analysis of particles after carbodiimide coupling of fluorescein onto amine terminated S13. c, Analysis of fluorescent particles after surface functionalization with hexadecyltrimethoxysilane to render the particles more hydrophobic.
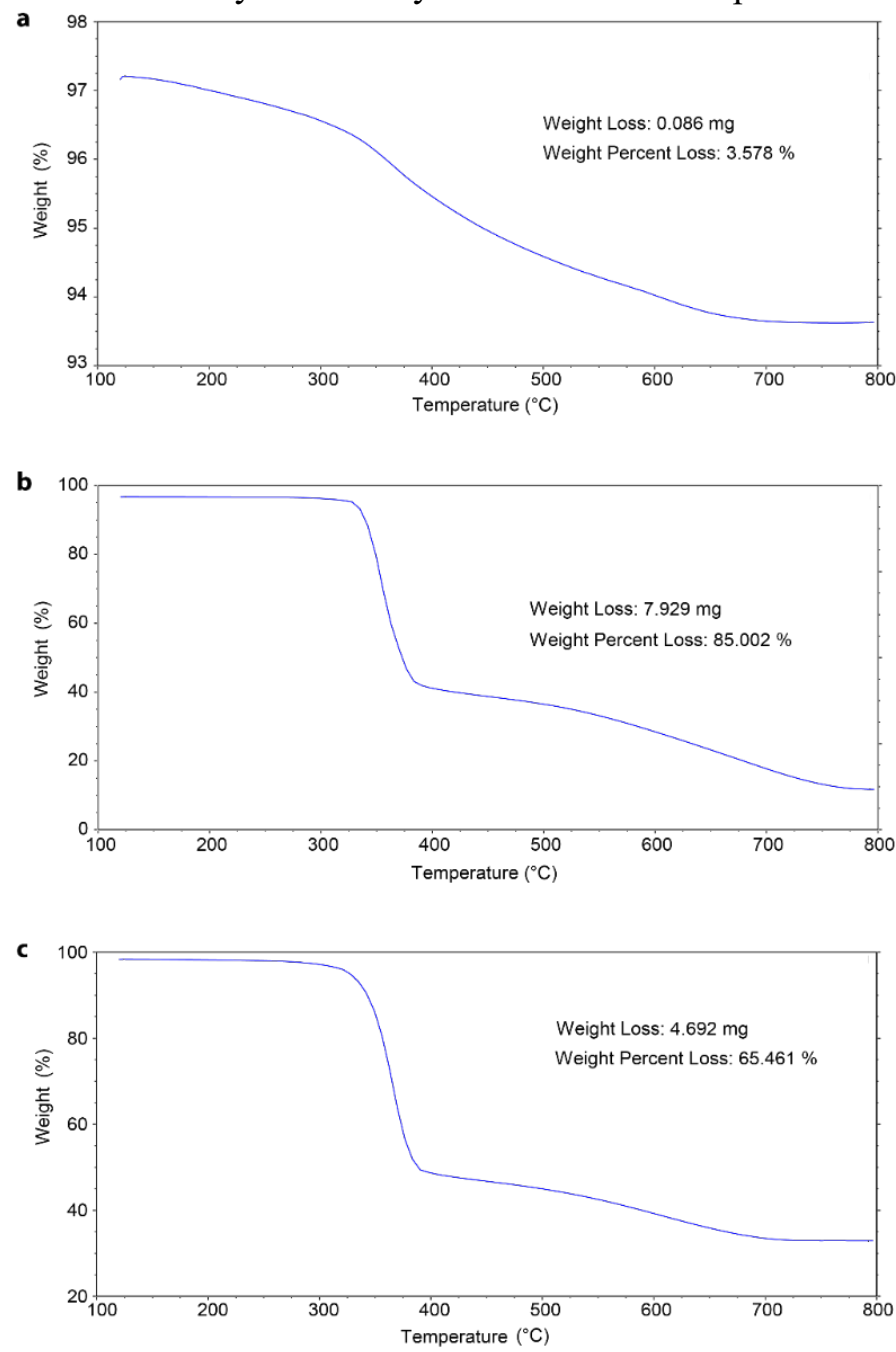
Table S1. Speeds of bromodecane droplets containing various silica (H13L) concentrations dispersed in different Triton X-100 concentrations were measured. For a given droplet, the highest speed reached during the imaging was assigned as the droplet's speed. For each experimental condition, at least 25 droplets were measured. This data is plotted in Figure 1.

\begin{tabular}{|ccc|ccc|ccc|}
\hline \multicolumn{2}{|c|}{$0.1 \mathrm{wt} \%$} & \multicolumn{3}{c|}{$0.5 \mathrm{wt} \%$ Triton X } & \multicolumn{3}{c|}{$1.0 \mathrm{wt} \%$ Triton X } \\
\hline Particle wt\% & $\begin{array}{c}\text { Speed } \\
(\mu \mathrm{m} / \mathrm{s})\end{array}$ & Stdv & Particle $\mathrm{wt} \%$ & $\begin{array}{c}\text { Speed } \\
(\mu \mathrm{m} / \mathrm{s})\end{array}$ & Stdv & $\begin{array}{c}\text { Particle } \\
\mathrm{wt} \%\end{array}$ & $\begin{array}{c}\text { Speed } \\
(\mu \mathrm{m} / \mathrm{s})\end{array}$ & Stdv \\
\hline 0.00 & 3.4 & 1.0 & 0.00 & 48.9 & 17.1 & 0.00 & 125.0 & 24.7 \\
0.05 & 74.8 & 12.7 & 0.05 & 167.56 & 21.8 & 0.05 & 231.5 & 17.2 \\
0.20 & 66.9 & 10.1 & 0.20 & 232.0 & 21.5 & 0.20 & 256.4 & 17.9 \\
0.40 & 53.6 & 15.4 & 0.40 & 233.6 & 12.3 & 0.40 & 285.3 & 30.2 \\
0.50 & 48.3 & 15.1 & 0.50 & 244.0 & 31.4 & 0.50 & 265.5 & 37.7 \\
0.60 & 49.8 & 11.1 & 0.60 & 243.9 & 22.9 & 0.60 & 280.5 & 22.5 \\
0.80 & 49.4 & 16.3 & 0.80 & 213.7 & 21.1 & 0.80 & 251.0 & 29.5 \\
1.00 & 49.8 & 3.1 & 1.00 & 166.0 & 35.6 & 1.00 & 234.4 & 29.5 \\
1.50 & 3.7 & 1.3 & 1.50 & 110.3 & 51.7 & 1.50 & 175.8 & 40.0 \\
2.00 & 4.1 & 1.8 & 2.00 & 48.9 & 40.8 & 2.00 & 159.8 & 52.5 \\
\hline
\end{tabular}

Table S2. Speeds of bromodecane droplets in various surfactant concentrations, with and without $1 \mathrm{wt} \%$ fluorescent silica particles. To keep particle surface coverage as constant as possible across all conditions, droplets with surface coverage in the range of $30 \%$ to $50 \%\left(\theta_{c}=66\right.$ to $\left.90^{\circ}\right)$ were used in these measurements. At least 6 droplets were measured and averaged for each condition. This data is plotted in Figure 5a.

\begin{tabular}{|lcccc|}
\hline Surfactant & \multicolumn{2}{c}{ Speed $(\mu \mathrm{m} / \mathrm{s})$} & \multicolumn{2}{c|}{ Speed $(\mu \mathrm{m} / \mathrm{s})$} \\
\hline & With Particles & $\mathrm{Stdv}$ & No Particles & $\mathrm{Stdv}$ \\
\hline $1 \mathrm{wt} \%$ SDS & 5.9 & 1.4 & 2.8 & 1.56 \\
$10 \mathrm{wt} \%$ SDS & 117.3 & 15.8 & 3.7 & 1.2 \\
$15 \mathrm{wt} \%$ SDS & 288.6 & 48.0 & 6.6 & 0.8 \\
$1 \mathrm{wt} \%$ SDS, $0.25 \mathrm{M} \mathrm{NaCl}$ & 345.9 & 74.4 & 11.2 & 5.1 \\
$1 \mathrm{wt} \%$ CTAB & 11.9 & 3.2 & 5.4 & 1.0 \\
$2.5 \mathrm{wt} \%$ CTAB & 189.5 & 41.45 & 15.5 & 10.2 \\
$1 \mathrm{wt} \%$ CTAB, $0.25 \mathrm{M} \mathrm{NaCl}$ & 251.6 & 42.9 & 16.9 & 8.0 \\
$0.5 \mathrm{wt} \%$ Triton X-100 & 256.9 & 29.5 & 48.9 & 17.1 \\
$0.5 \mathrm{wt} \%$ Triton X-100, 0.25 M NaCl & 278.9 & 23.9 & 41.9 & 15.4 \\
$1 \mathrm{wt} \%$ Triton X-100 & 351.8 & 48.8 & 125.0 & 24.7 \\
\hline
\end{tabular}


Table S3. Speeds of various brominated oil droplets with and without $1 \mathrm{wt} \%$ fluorescent silica particles in $0.5 \mathrm{wt} \%$ Triton X-100. To keep particle surface coverage as constant as possible across all conditions, droplets with particle surface coverage in the range of $30 \%$ to $50 \%\left(\theta_{c}=66\right.$ to $\left.90^{\circ}\right)$ were used in these measurements. At least 6 droplets were measured and averaged for each condition. This data is plotted in Figure 5b.

\begin{tabular}{|lcccc|}
\hline Brominated oil & \multicolumn{2}{c}{ Speed $(\mu \mathrm{m} / \mathrm{s})$} & \multicolumn{2}{c|}{ Speed $(\mu \mathrm{m} / \mathrm{s})$} \\
\hline & With Particles & Stdv & No Particles & Stdv \\
\hline Brominated vegetable oil & 9.2 & 3.9 & 2.5 & 0.5 \\
Bromohexadecane & 99.3 & 24.7 & 4.4 & 2.1 \\
Bromododecane & 241.3 & 14.8 & 69.7 & 12.0 \\
Bromodecane & 256.9 & 29.5 & 48.9 & 17.1 \\
Bromooctane & 223.5 & 23.8 & 12.4 & 5.8 \\
\hline
\end{tabular}

Table S4. Solubilization rates of various brominated oils in Triton X-100 and bromodecane in various surfactants. Solubilization rates were quantified by measuring the change in droplet diameter over an hour in each surfactant condition. Surfactants labeled with an * indicate that the solution was supersaturated and the solution was handled carefully to not induce crystallization and no significant crystallization occurred during the measurement. Each measurement was collected for one droplet. Although we could not measure a significant change in the bromohexadecane droplet diameter within one hour, literature ${ }^{1}$ indicates that hexadecane does solubilize in Triton X-100; we thus believe that the bromohexadecane is still solubilizing, just slowly. In comparison, we expect the brominated vegetable oil, which is highly viscous, solubilizes at a slower rate than the bromohexadecane.

\begin{tabular}{|llc|}
\hline Brominated oil & Surfactant concentration & Solubilization rate $(\mu \mathrm{m} / \mathrm{min})$ \\
\hline 1-Bromodecane & $1 \mathrm{wt} \%$ SDS & 0.035 \\
1-Bromodecane & $5 \mathrm{wt} \%$ SDS & 0.095 \\
1-Bromodecane & $1 \mathrm{wt} \%$ SDS with $0.25 \mathrm{M} \mathrm{NaCl}$ & 0.091 \\
1-Bromodecane & $5 \mathrm{wt} \%$ SDS with $0.25 \mathrm{M} \mathrm{NaCl} *$ & 0.112 \\
1-Bromodecane & $1 \mathrm{wt} \%$ CTAB $*$ & 0.068 \\
1-Bromodecane & $2.5 \mathrm{wt} \%$ CTAB * & 0.163 \\
1-Bromodecane & $1 \mathrm{wt} \%$ CTAB with $0.25 \mathrm{M} \mathrm{NaCl}$ & 0.131 \\
1-Bromodecane & $2.5 \mathrm{wt} \%$ CTAB with $0.25 \mathrm{M} \mathrm{NaCl}$ & 0.424 \\
1-Bromooctane & $0.5 \mathrm{wt} \%$ Triton X-100 & 0.412 \\
1-Bromodecane & $0.5 \mathrm{wt} \%$ Triton X-100 & 0.237 \\
1-Bromododecane & $0.5 \mathrm{wt} \%$ Triton X-100 & 0.136 \\
1-Bromohexadecane & $0.5 \mathrm{wt} \%$ Triton X-100 & $\leq 0.01$ \\
Brominated vegetable oil & $0.5 \mathrm{wt} \%$ Triton X-100 & $<0.01$ \\
\hline
\end{tabular}

Video S1. Video of bromodecane droplets with $0.5 \mathrm{wt} \% \mathrm{H} 13 \mathrm{~L}$ particles in $0.1 \mathrm{wt} \%$ Triton $\mathrm{X}-100$. Video is played back in 6x speed.

Video S2. Video of bromodecane droplets containing $2 \mathrm{wt} \%$ fluorescent particles dispersed $0.5 \mathrm{wt} \%$ Triton X-100 immediately after preparation by vortex mixing and added to an imaging chamber containing surfactant. The particles are initially dispersed inside the droplets, but over time, the particles accumulate at the droplet surface and aggregate in a cap at the rear pole of the droplet. Droplets begin to move when the interfacial particle cap forms. Video is played back in 2x speed. 
Video S3. Video of bromodecane droplets with $2 \mathrm{wt} \%$ fluorescent particles dispersed in $0.5 \mathrm{wt} \%$ Triton X100 . Video is played back in $2 \mathrm{x}$ speed.

Video S4. Bromodecane with $0.5 \mathrm{wt} \%$ fluorescent particles in $2.5 \mathrm{wt} \% \mathrm{CTAB}$. Video is played back in $2 \mathrm{x}$ speed.

1. Zhong, H. et al. Aggregate-based sub-CMC solubilization of hexadecane by surfactants. RSC Adv. 5, 78142-78149 (2015). 